\title{
Diffracted diffraction radiation and its application to beam diagnostics
}

\author{
Yu.A. Goponov ${ }^{\text {a }}$, R.A. Shatokhin ${ }^{\text {a }}$, K. Sumitani ${ }^{\mathrm{b}}$, V.V. Syshchenko ${ }^{\text {a }}$, Y. Takabayashi ${ }^{\mathrm{c}}$, \\ I.E. Vnukov ${ }^{\mathrm{a}, *}$ \\ a Belgorod National Research University, Belgorod, Russia \\ b Japan Synchrotron Radiation Research Institute (JASRI), 1-1-1 Kouto, Sayo-cho, Sayo-gun, Hyogo 679-5198, Japan \\ c SAGA Light Source, 8-7 Yayoigaoka, Tosu, Saga 841-0005, Japan
}

\section{A R T I C L E I N F O}

\section{Keywords:}

Beam diagnostics

Diffracted diffraction radiation

Linear collider

Angular divergence

Crystal

$\mathrm{X}$-ray mirror

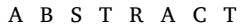

We present theoretical considerations for diffracted diffraction radiation and also propose an application of this process to diagnosing ultra-relativistic electron (positron) beams for the first time. Diffraction radiation is produced when relativistic particles move near a target. If the target is a crystal or X-ray mirror, diffraction radiation in the X-ray region is expected to be diffracted at the Bragg angle and therefore be detectable. We present a scheme for applying this process to measurements of the beam angular spread, and consider how to conduct a proof-of-principle experiment for the proposed method.
\end{abstract}

(C) 2017 Elsevier B.V. All rights reserved.

\section{Introduction}

Beam size and angular divergence are among the most important parameters for particle accelerators, and many beam diagnostic methods have been developed for measuring these characteristics. For electron linear accelerators, one conventional method is to use optical radiation, such as fluorescent light, optical transition radiation (OTR) [1], optical diffraction radiation (ODR) [2], or Smith-Purcell radiation [3]. However, it was recently found that OTR cannot be used to measure the profiles of electron beams at linear accelerators designed for use as X-ray free electron lasers (XFELs) because OTR becomes coherent when the bunch duration of the beam is sufficiently small [4] compared to the wavelength of the measured photons. A similar difficulty is expected to occur when the transverse beam size is extremely small, e.g., for proposed electron-positron linear colliders such as the International Linear Collider (ILC) [5] and the Compact Linear Collider (CLIC) [6].

To prevent coherence, photons with shorter wavelengths are required. Some years ago, the use of radiation in the X-ray region, socalled parametric X-ray radiation (PXR), was proposed [7,8]. Recently, proof-of-principle experiments on beam profile measurements using PXR have been performed at the SAGA Light Source [8,9] and Mainz Microtron [10]. For relativistic electrons, PXR is always accompanied by crystal-diffracted radiation, which is created directly inside the target as diffracted bremsstrahlung radiation or on its surface as diffracted transition radiation (DTR) [11].
Beam diagnostic methods using DTR have been proposed by a number of researchers [12-14]. We first proposed the use of DTR in a thin crystal to perform beam diagnostics for linear colliders [13]. The proposed method is based on the following two considerations. First, for linear colliders, the beam angular divergence is not so small (on the order of tens of $\mu \mathrm{rad}$ ), although the beam size at the interaction point is extremely small (on the order of $\mathrm{nm}$ ) $[5,6]$. Therefore, the beam angular divergence is easier to measure than the beam size. The beam size $\sigma$ can be estimated from the beam angular divergence $\theta_{e}$ and the beam emittance $\epsilon$ using $\epsilon \approx \sigma \times \theta_{e}$. The beam emittance can be obtained from calculation or measurements at the earlier stage of acceleration. In comparison with Ref. [13] here we use approximate equality because of possible magnetic systems chromaticism and non-linearity. In any case this approach gives us possibility to obtain information about changing of the electron beam size and divergence in the acceleration process. Second, the angular distribution of DTR is primarily determined by the angular distribution of the incident beam because, for linear colliders, a beam angular divergence of about 20-40 $\mu \mathrm{rad}$ [5] is sufficiently larger than the characteristic angle of the DTR angular distribution, which is $1 / \gamma$ (where $\gamma$ is the Lorentz factor). We have previously demonstrated that PXR is not suitable for measuring the beam angular divergence because the characteristic angle of PXR is too large compared to the beam angular divergence [13].

The main drawback for using DTR in a thin crystal for beam diagnostics is that the crystal is destroyed when struck by an intense

\footnotetext{
* Corresponding author.

E-mail address: vnukov@bsu.edu.ru (I.E. Vnukov).
} 
electron beam (e.g., [15]). To circumvent these problems, we have proposed the use of surface emissions from a crystal when a fast particle moves near it [13]. This so-called surface PXR [16], an analogue of DTR, arises because of differences between the particle field in a vacuum and a dense medium. We previously referred to this type of emission as surface DTR. However, in accordance with physics tradition, the correct term for an emission that arises when a fast particle moves near a target edge without intersecting it is diffraction radiation (DR) [17]. Therefore, herein we use the term diffracted diffraction radiation (DDR) rather than surface DTR.

From research involving electron beams and targets, DR has been known for more than 50 years, and is widely used for generation of microwaves using fast electrons. Twenty years ago, optical DR from metal foils was observed experimentally [18] and soon began to be used for electron beam diagnostics; see, for example, Refs. [2,17] and references therein. Recently [19], it was shown that Smith-Purcell radiation [20], which is a well-known manifestation of ODR in a metal target with a specific shape or in a stack of conducting foils separated by non-conducting gaps, may be observed at X-ray wavelengths in stacks of dielectric foils with vacuum gaps. Smith-Purcell radiation arises due to constructive interference of radiation generated by relativistic electrons in different conducting [20] or dielectric [19] foils and in X-ray region is similar surface PXR [16]. DTR and DDR are result of diffraction of emission arising on the inlet side of the crystal when a relativistic particle moves across the target or near it.

Both TR and DR have the same physical nature and are different manifestations of so-called polarization radiation. Both situations involve emissions from electron shells in the atoms of a medium induced by the field of a moving charged particle. Therefore, for small distances between the relativistic charged particle and a target, DR can also be expected at X-ray wavelengths. It is clear that the emission occurs in the target because all types of polarization emissions are generated only by atoms in the medium. If the target is a thin crystal, diffraction can be expected at Bragg angles, producing detectable peaks.

Based on the above discussion and the obvious advantages of using $\mathrm{X}$-ray emission caused by electrons moving near thin crystals to diagnose ultra-high energy electron beams, it is important to investigate the possibility of non-destructive beam parameter estimation by means of the DDR angular distribution measurement.

\section{Theoretical considerations}

It has been reported that for electrons with an energy of several tens of $\mathrm{GeV}$, the angular density of emission intensity at the center of the diffraction spot is much lower for PXR with a dip width of several mrad for this observation angle than for DTR with the characteristic emission angle about $\gamma^{-1}$ concentrated near the Bragg's direction [13,14,21]. Because of the same nature of volume and surface emissions, we may expect a similar relationship between surface PXR and DDR. Therefore, we can ignore the surface PXR contribution and focus only on DDR.

In the absence of an exact theory describing DDR in the X-ray region, we used an eikonal approximation [19] to describe the diffraction and transition radiation in this region. The spectral-angular distribution of the radiation due to the interaction of a moving particle with a semiinfinite dielectric plate of thickness $a$ (see Fig. 1) can be written as:

$\frac{d^{2} I}{d \omega d \Omega}=\frac{e^{2} \gamma^{2}}{2 \pi^{2}}\left\{1-\cos \left[\frac{\omega_{\mathrm{p}}^{2}}{\omega^{2}} \frac{\pi a}{\lambda}\right]\right\} F\left(\omega, \theta_{x}, \theta_{y}\right)$,

where $\theta_{x}$ and $\theta_{y}$ are respectively the photon emission angle in the horizontal and vertical planes with respect to the direction of electron motion, $\omega$ is the photon frequency, $\gamma$ is the Lorentz factor, $\omega_{p}$ is the plasma frequency for the medium, and $\lambda$ is the emission wavelength.

To describe the angular distribution of the emission energy $\hbar \omega$, which is dependent on the impact parameter $x_{0}$ when the particle moves

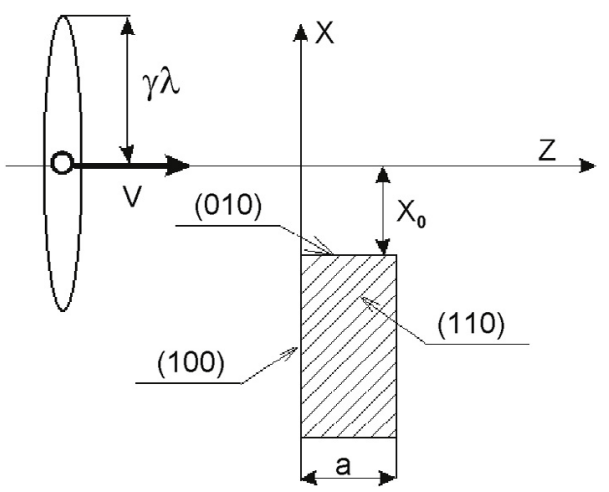

Fig. 1. Particle motion near the edge of the target plate. $\gamma \lambda$ is the characteristic transverse dimension of the Fourier component of the particle's Coulomb field corresponding to the frequency $\omega$ [19].

near the plate, that is, for a negative $x_{0}$, the function $F\left(\omega, \theta_{x}, \theta_{y}\right)$ is written as:

$F\left(\omega, \theta_{x}, \theta_{y}\right)=\frac{1+2 \gamma^{2} \theta_{y}^{2}}{\left(1+\gamma^{2} \theta_{y}^{2}\right)\left(1+\gamma^{2} \theta^{2}\right)} \exp \left(-\frac{4 \pi\left|x_{0}\right|}{\gamma \lambda} \sqrt{1+\gamma^{2} \theta_{y}^{2}}\right)$.

For a large positive $x_{0}, F\left(\theta_{x}, \theta_{y}\right)$ is similar to the known formula for the TR angular distribution [19]. Similar expressions for the DR angular distribution are given in Ref. [17]. The expression (2) is obtained in the approximation when a particle's field in a medium is coincided with non-disturbed Coulomb field of the uniformly moving particle in vacuum and therefore does not include the plasma frequency [19]. Similar approach was used in Ref. [14] for DTR description in thin crystals and high electron energy. This approach is valid because we are interesting in the photon energy region $\hbar \omega \ll \gamma \hbar \omega_{p} \sim$ some $\mathrm{MeV}$, where the TR and DR angular distribution and intensity are not practically depend on the medium parameters, see, for example, [17].

Eqs. (1) and (2) describe the angular distribution of the emission from a thin plate and include a term that accounts for radiation interference from the front and back surfaces of the plate. Therefore, the angular distribution of the DR arising on the front surface of the target may be written as:

$\frac{d^{2} I_{\mathrm{DR}}}{d \omega d \Omega}=\frac{e^{2} \gamma^{2}}{2 \pi^{2}} \frac{1+2 \gamma^{2} \theta_{y}^{2}}{\left(1+\gamma^{2} \theta_{y}^{2}\right)\left(1+\gamma^{2} \theta^{2}\right)} \exp \left(-\frac{4 \pi\left|x_{0}\right|}{\gamma \lambda} \sqrt{1+\gamma^{2} \theta_{y}^{2}}\right)$.

As discussed above, if the target is a crystal, this emission may be diffracted at the Bragg angle. Since DR is similar to TR, the angular distribution of the DDR at the center of the reflex may be written as:

$Y_{\mathrm{DDR}}(\theta)=\int d \hbar \omega \int \frac{d^{2} I_{\mathrm{DR}}^{*}}{d \hbar \omega d \Omega} R\left(\hbar \omega, \vec{n}, \vec{g}, \Theta_{\mathrm{D}}\right) S^{*}(\hbar \omega, \vec{n}, T) d \Omega$,

where $\vec{n}$ is a vector corresponding to an emitted photon (with energy $\hbar \omega$ and momentum $\vec{k}$ ); $\vec{g}$ is the reciprocal lattice vector; $d^{2} I_{\mathrm{DR}}^{*} / d \hbar \omega d \Omega$ is the DR angular distribution, taking into account the divergence of the primary electron beam; $R\left(\hbar \omega, \vec{n}, \vec{g}, \Theta_{\mathrm{D}}\right)$ is the reflectivity for the direction vectors $\vec{n}$ and $\vec{g}$, as determined by the crystallographic plane orientation with respect to the electron beam direction $\Theta$ and the detector location $\Theta_{\mathrm{D}} ; S^{*}(\hbar \omega, \vec{n}, T)$ is a function taking into account photon absorption in the crystal and the geometry of the experiment; and $T$ is the crystal thickness [22]. The integration in Eq. (4) is performed over all angles and energies for photons striking the collimator.

This approach allows us to describe the contribution of the diffracted photons, such as bremsstrahlung and TR, in experimentally measured yields of electron emissions at Bragg angles with an error less than $20 \%$ [23]. 


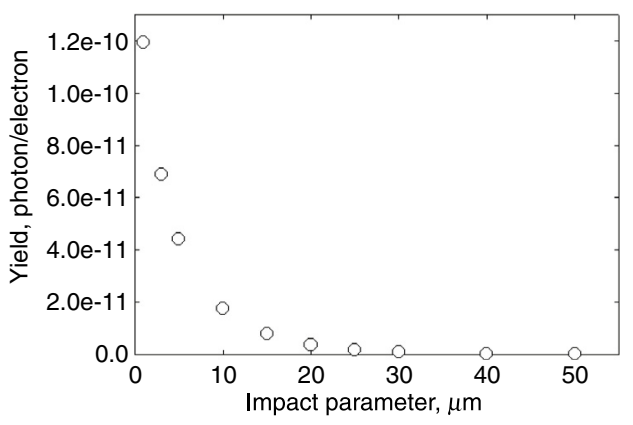

Fig. 2. Dependence of the maximum emission yield on the impact parameter.

\section{Calculation results and discussion}

The expressions presented above for the diffracted radiation angular distribution are applicable to a situation in which the particle movement direction is normal to the plate. Therefore, to calculate the DDR characteristics, we chose a case of so-called extremely asymmetric diffraction, in which the particle field hits the crystal normal to its surface and diffracted photons are emitted through a lateral face [16]. This situation is realized for a crystal with a cubical structure where all target faces coincide with (100) type crystallographic planes. In this case, photons moving along the (010) plane and normal to the (100) face are diffracted on (110) planes by $45^{\circ}$ with respect to the photon direction. They then exit the crystal through the lateral face (see Fig. 1) and can be registered by a detector placed at an angle of $90^{\circ}$.

To compare the DTR and DDR for the purpose of developing electron beam diagnostics, most of the calculations were done for conditions similar to those previously reported [13]. An electron beam with an energy of $200 \mathrm{GeV}$ was incident on a silicon crystal with a thickness of 20 $\mu \mathrm{m}$. The (220) reflection was investigated with a photon energy of $\hbar \omega \sim$ $4.566 \mathrm{keV}$. The parameter $\gamma \lambda$ was about $106 \mu \mathrm{m}$. The target thickness was greater than the characteristic length of photons diffraction process in the crystal $l_{\text {ext }} \approx 1.87 \mu \mathrm{m}$ for this reflection order and the photon energy, see Refs. [22,23] and reference therein. The detection system was located $10 \mathrm{~m}$ from the crystal at an angle of $\Theta_{\mathrm{D}}=2 \Theta_{\mathrm{B}}=90^{\circ}$. The square detector, the size of which was $0.01 \times 0.01 \mathrm{~mm}^{2}$, was moved through the reflex center in $0.01 \mathrm{~mm}$ steps. The expressions obtained in Ref. [19] do not take into account the position where the photons are produced; therefore, the calculation procedure ignored the process of photon absorption. To match the ILC and CLIC conditions, the electron beam spatial size was less than $1 \mu \mathrm{m}$ and the beam angular divergence was far greater than $\gamma^{-1}$; therefore, the electron beam spatial distribution and polarization of the emission were also ignored.

Fig. 2 shows the dependence of the emission yield in the top of the diffracted radiation angular distribution (see below) on the impact parameter. The calculation was made for an electron beam divergence of $40 \mu \mathrm{rad}$ and $20 \mu \mathrm{rad}$ for the horizontal and vertical planes, respectively.

It can be seen that increasing the impact parameter rapidly reduces the emission yield because of the exponential term in the formula for the emission yield contribution [see Eq. (3)]. For further calculation, we chose $x_{0}=10 \mu \mathrm{m} \approx 0.1 \gamma \lambda$, because for lower values, the electron beam moves too close to the crystal, and for larger values, the emission yield becomes too small. Fig. 3 shows the dependence of the intensity maximum value on the electron energy in the range $50-250 \mathrm{GeV}$.

From the figure, it can be seen that decreasing the electron energy strong suppresses the yield because $\gamma \lambda$ decreases, which is connected with the strong contribution of the exponential term to the emission yield. It should be remarked that for large values of electron beam divergence $\left(\theta_{e} \gg \gamma^{-1}\right)$, the DDR angular distribution shape in the investigated energy region remains approximately the same.

Fig. 4 shows the influence of the electron beam divergence in both planes on the emission yield angular distribution. The electron

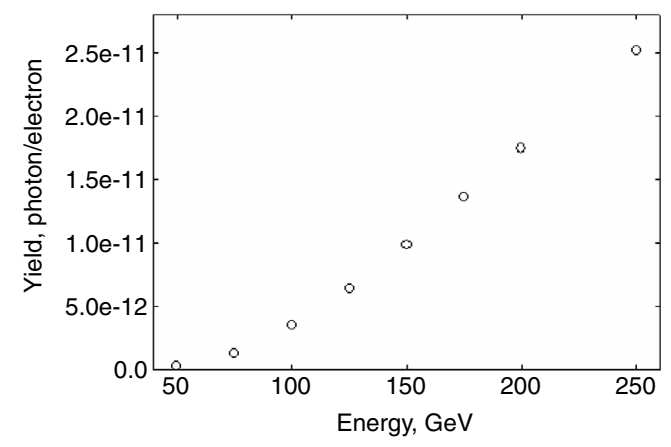

Fig. 3. Dependence of the maximum emission yield on the electron energy.

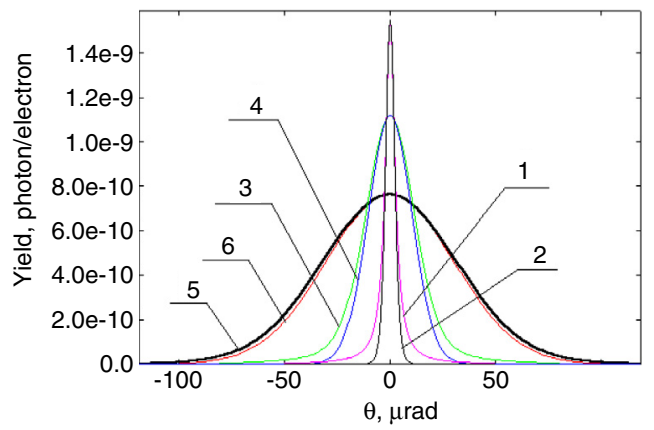

Fig. 4. Emission angular distribution in both planes for different electron beam divergences. Curves 1 and 2 are calculated for a divergence of $1 \mu \mathrm{rad}\left(<\gamma^{-1} \sim 2.6 \mu \mathrm{rad}\right)$. Curves 3 and 4 are calculated for a divergence of $10 \mu \mathrm{rad}$, and curves 3 and 4 for a divergence of $30 \mu \mathrm{rad}$. Odd-numbered curves are horizontal distributions, and even-numbered curves are vertical distributions.

beam divergence in both planes was assumed to be the same. From the figure, it is seen that the vertical distribution is narrower than the horizontal distribution. Because they are connected by the square root in the exponential term, large vertical angles of the radiation are suppressed according to the electron movement direction (see Fig. 4 and its explanation).

The dependence of $\sigma_{\text {calc }}$, which is the standard deviation in the DDR angular distribution based on the detector angular acceptance $\vartheta_{\mathrm{c}}=$ $1 \mu \mathrm{rad}$ ), on the beam divergence $\theta_{e}$ is shown in Fig. 5(a) by blue circles and red triangles for the vertical and horizontal planes, respectively. Fig. 5(b) shows the dependence of the $\sigma_{\text {calc }} / \theta_{e}$ ratio on $\theta_{e}$. As above, the calculations were performed for the same divergences in both planes.

It can be seen that for comparatively large vertical divergence values, $\sigma_{\text {calc }}$ practically coincides with the beam divergence. This is due to the influence of the square root term in the exponent of Eq. (3). The contribution of photons with large angles in the vertical plane to the DR yield is practically negligible. Therefore, the diffracted emission angular distribution in the vertical plane reproduces the electron beam angular distribution.

For the horizontal plane, we have a slightly different situation. For small electron beam divergence values, the contribution of the self-emission angular distribution to the measured radiation angular distribution is comparatively large; therefore, $\sigma_{\text {calc }}$ is greater than the divergence value. For large electron beam divergences, the influence of the emission angular distribution becomes smaller and the difference between $\sigma_{\text {calc }}$ and the electron beam divergence becomes negligible.

The calculations showed that the beam divergence in one plane does not appreciably influence the $\sigma_{\text {calc }}$ value in the other plane. Specifically, changing $\sigma_{\text {calc }}$ for a fixed electron beam divergence in one plane affected the value of the beam divergence in the other plane by less than $1 \%$.

From the information presented above, it is clear that using DDR for high-energy electron beam diagnostics instead of DTR [13] provides 

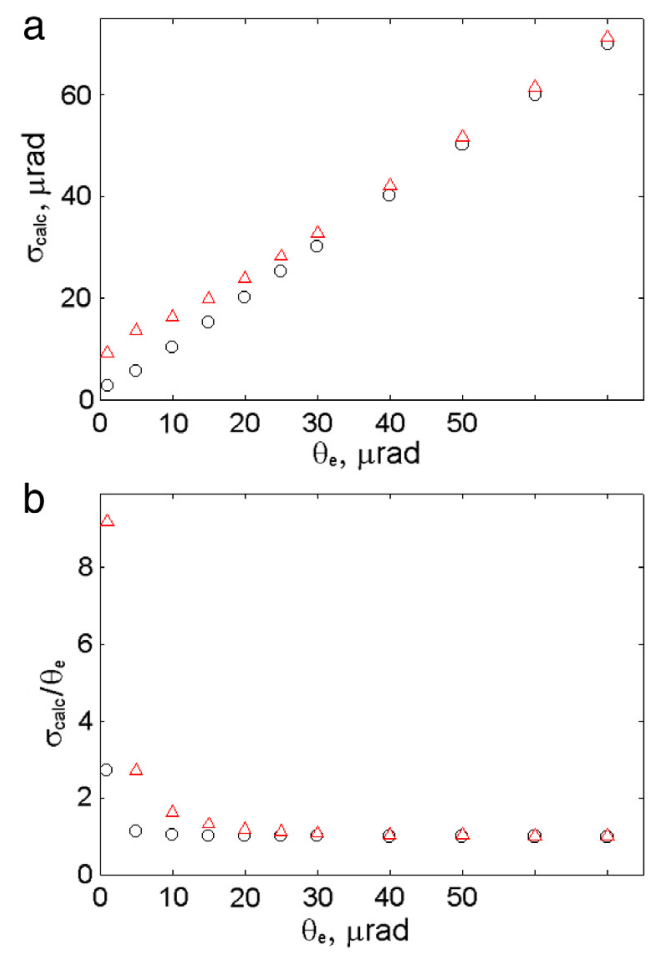

Fig. 5. Dependence of $\sigma_{\text {calc }}$ on the electron beam divergence $\theta_{e}$ : (a) $\sigma_{\text {calc }}$ value and (b) $\sigma_{\text {calc }} / \theta_{e} . \circ=$ vertical plane; $\triangle=$ horizontal plane.

better accuracy and avoids damage to the crystal target. The main disadvantage of DDR for high-energy electron beam diagnostics is the low intensity associated with exponential suppression of the radiation yield when the impact parameter increases, due to the short wavelength.

PXR and DTR can be produced not only by crystals, where the lattice parameter $d$ limits the wavelength range of the generated radiation to $\lambda<2 d$, but also by artificial periodic structures with an arbitrary lattice parameter, which are often called multi-layered X-ray mirrors [24,25]. Increasing the lattice parameter up to several tens of nanometers will increase $\gamma \lambda$ to several millimeters and allow us to measure linear collider electron beam parameters in the particle energy region of $20-500 \mathrm{GeV}$. See, for example, Ref. [25], where the authors have confidently observed PXR and especially DTR with energy of several hundred electron volts from a $\mathrm{Cr} / \mathrm{Sc}$ multilayer radiator. Moreover, DDR in such X-ray mirrors could be used for diagnostics of electron beams from XFEL linear accelerators with particle energies of $10-20 \mathrm{GeV}$. X-ray mirrors with similar or greater lattice parameters may be used on existing electron accelerators with energies of about $1 \mathrm{GeV}$ to verify the presence of DDR and investigate its characteristics, so that measurement results can be compared with theoretical results for later use in determining the beam parameters for future colliders and XFEL linear accelerators.

A proof-of-principle experiment on the use of DDR could be carried out at the KEK Accelerator Test Facility with an electron energy of 1.3 $\mathrm{GeV}$, where the beam size is controllable in the range from several tens of nanometers to a few micrometers [26]. Because of the presence of a magnet that deflects the electron beam after interaction with the laser photons, and the detector that registers $\gamma$-rays caused by laser Compton scattering in this experiment, such a technique would allow aiming the electron beam on the target and registering electrons passing through the X-ray mirror, as in the experiment in Ref. [18], which aimed at detecting ODR from relativistic electrons.

The most optimal way is using a $\mathrm{Cr} / \mathrm{Sc}$ multilayer radiator with the lattice parameter $d=2.34 \mathrm{~nm}$ turned on the angle $\Theta_{B}=45^{\circ}$ as in the experiment [25]. Similar mirrors with 250 layers provide a reflection coefficient about $10 \%,[27]$ and angular size of the reflection spot $\Delta \theta=$
$\Delta \omega / \omega \cdot \operatorname{tg}\left(\Theta_{B}\right) \approx 4 \mathrm{mrad}$. As for zero impact parameter transition radiation intensity and diffraction radiation one have approximately the same order of magnitude [17] we may estimate transition radiation yield. For the photon energy $\hbar \omega=376 \mathrm{eV}$, energy resolution $\Delta \omega / \omega \sim 0.004$ and wave length $\lambda=3.286 \mathrm{~nm}$ expected photon yield $N \approx \frac{\alpha}{\pi} \ln \frac{\omega_{p}^{2} \gamma^{2}}{\omega_{1} \omega_{2}} \ln \frac{\omega_{2}}{\omega_{1}}$ (see Eq. 1.76 in the Ref. [28]) is about $10^{-5}$ photon/electron. Here $\alpha$ is fine structure constant, $\omega_{1}$ and $\omega_{2}$ are the borders of the photon energy range.

For the impact parameter $x_{0}=0.8 \mu \mathrm{m} \sim 0.1 \gamma \lambda=8.36 \mu \mathrm{m}$ and reflection coefficient $10 \%$ we may wait diffraction radiation yield in whole cone about $10^{-7}$ photon/electron. Decreasing of the collimation angle up to $1 \mathrm{mrad}$ and using a detector with efficiency of $10 \%$ reduce this value up to $10^{-9}$ events/electron. For the number of electron in a bunch about $10^{10}$ needful statistics may be obtained during some hours. Using of $\mathrm{Mo} / \mathrm{Be}$ or $\mathrm{Mo} / \mathrm{Si} \mathrm{X}$-ray mirrors with wave length about $15 \mathrm{~nm}$ may increase the $\gamma \lambda$ value and the reflection coefficient up to $40 \mu \mathrm{m}$ and $60 \%$ respectively [27]. Unfortunately these X-ray mirrors have the energy resolution about some percent; therefore we will obtain an increasing of the angular size of the reflection spot about ten times.

As discussed above, the DR angular intensity distribution used in the calculation was for the case of particles normally incident on the crystal and, therefore, it is not valid for X-ray mirrors because of their grating structure. The X-ray diffraction calculation method [22] is based on the theory of X-ray diffraction in a perfect crystal [29] and cannot be exactly transferred to X-ray mirrors. Possible imperfections in the X-ray mirror structure must also be taken into account (see, e.g., Ref. [25]). The last item is especially important for comparing experimental and calculation results. The resolution to these problems is under investigation and will be presented elsewhere.

\section{Summary and conclusions}

The main disadvantage of performing high-energy electron beam diagnostics by measuring the DTR angular distribution in a thin crystal [13] is that striking a thin crystal target with a full-intensity beam will destroy it. To circumvent this problem, we have proposed the use of DDR, and have demonstrated that it can be applied as a new diagnostic method for an electron (positron) beam at future linear colliders. Because the beam angular divergence is significantly larger than the characteristic angle of DDR, the angular distribution of DDR is mainly determined by that of the incident beam; therefore, the beam angular divergence can be derived from the DDR angular distribution. While the Shintake monitor measures beam size [30], our proposed method measures beam angular divergence. Therefore, the proposed method may prove useful as a complementary beam monitor for linear colliders. In contrast to the Shintake monitor, the proposed method has the following advantages: (1) the experimental setup can be installed at a straight section of the accelerator, i.e., a bending magnet is unnecessary; (2) it is low in cost; and (3) multiple monitors may be installed.

A serious problem for electron beam diagnostics using DDR is that the emission yield is too small. The yield is associated with the exponential term in the expression for the DR intensity angular distribution, and we may observe this radiation confidently only for very small values of the impact parameter. We have proposed to solve this problem by using multi-layered X-ray mirrors instead of crystals. In this case, possible values of impact parameters are expected to be higher and observation of the effect should be simpler. This radiation process in crystals and $\mathrm{X}$-ray mirrors has not yet been observed experimentally; therefore, its confirmation is necessary. As discussed, confirmation may be possible using a $1 \mathrm{GeV}$ class electron beam. A detailed consideration of DDR from an X-ray mirror will be published elsewhere.

\section{Acknowledgment}

This work was supported in part by JSPS KAKENHI Grant Number JP26400304. 


\section{References}

[1] R.B. Fiorito, in: Proceedings of PAC09, 2009, pp. 741.

[2] J. Urakawa, H. Hayano, K. Kubo, S. Kuroda, N. Terunuma, M. Kuriki, T. Okugi, T. Naito, S. Araki, A. Potylitsyn, G. Naumenko, P. Karataev, N. Potylitsyna, I. Vnukov, T. Hirose, R. Hamatsu, T. Muto, M. Ikezawa, Y. Shibata, Nucl. Instrum. Methods A 472 (2001) 309.

[3] G. Kube, H. Backe, W. Lauth, H. Schöpe, in: Proceedings of DIPAC2003, 2003, pp. 40.

[4] H. Loos et al., in: Proceedings of FEL08, 2008, pp. 485.

[5] ILC Technical Design Report, 2013.

[6] A Multi-TeV Linear Collider Based on CLIC Technology: CLIC Conceptual Design Report, 2012.

[7] A. Gogolev, A. Potylitsyn, G. Kube, J. Phys. Conf. Ser. 357 (2012) 012018.

[8] Y. Takabayashi, Phys. Lett. A 376 (2012) 2408.

[9] Y. Takabayashi, K. Sumitani, Phys. Lett. A 377 (2013) 2577.

[10] G. Kube, C. Behrens, A.S. Gogolev, Yu.P. Popov, A.P. Potylitsyn, W. Lauth, S. Weisse, in: Proceedings of IPAC2013, 2013, pp. 491.

[11] K.-H. Brenzinger, C. Herberg, B. Limburg, et al., Z. Phys. A 358 (1997) 107.

[12] V.V. Kaplin, S.R. Uglov, Nucl. Instrum. Methods B 145 (1998) 253.

[13] Yu.A. Goponov, M.A. Sidnin, K. Sumitani, Y. Takabayashi, I.E. Vnukov, Nucl. Instrum. Methods A 808 (2016) 71.

[14] I. Chaikovska, R. Chehab, X. Artru, A.V. Shchagin, Nucl. Instrum. Methods B 402 (2017) 75.

[15] A.A. Babaev, A.S. Gogolev, J. Phys. Conf. Ser. 732 (2016) 012030.

[16] A.I. Benediktovitch, I.D. Feranchuk, J. Phys. Conf. Ser. 236 (2010) 012015.
[17] A.P. Potylitsyn, M.I. Ryazanov, M.N. Strikhanov, A.A. Tishchenko, Diffraction Radiation From Relativistic Particles, Springer, Heidelberg, 2011.

[18] I.E. Vnukov, B.N. Kalinin, G.A. Naumenko, D.V. Padalko, A.P. Potylitsyn, O.V. Chefonov, JETP Lett. 67 (1998) 802.

[19] V.V. Syshchenko, N.F. Shulga, J. Surf. Invest.: X-Ray Synch. Neutron Tech. 5 (2011) 392.

[20] S.J. Smith, E.M. Purcell, Phys. Rev. 92 (1953) 1069.

[21] Yu.A. Goponov, S.A. Laktionova, O.O. Pligina, M.A. Sidnin, I.E. Vnukov, Nucl. Instrum. Methods B 355 (2015) 150.

[22] S.A. Laktionova, O.O. Pligina, M.A. Sidnin, I.E. Vnukov, J. Phys. Conf. Ser. 517 (2014) 012020

[23] Yu.A. Goponov, S.A. Laktionova, M.A. Sidnin, I.E. Vnukov, Nucl. Instrum. Methods B 402 (2017) 92.

[24] N.N. Nasonov, V.V. Kaplin, S.R. Uglov, V.N. Zabaev, M.A. Piestrup, C.K. Gary, Nucl. Instrum. Methods B 227 (2005) 41.

[25] S.R. Uglov, V.V. Kaplin, A.S. Kubankin, J.-M. André, K. Le Guen, Ph. Jonnard, S. de Rossi, E. Meltchakov, F. Delmotte, J. Phys. Conf. Ser. 732 (2016) 012017.

[26] J. Yan, Y. Yamaguchi, Y. Kamiya, S. Komamiya, M. Oroku, T. Okugi, N. Terunuma, K. Kubo, T. Tauchi, J. Urakawa, Nucl. Instrum. Methods A 740 (2014) 131.

[27] M.M. Barysheva, A.E. Pestov, N.N. Salashchenko, M.N. Toropov, N.I. Chkhalo, Phys.Usp. 55 (2012) 681.

[28] G.M. Garibian, C. Yang, X-Ray Transition Radiation, Akad. Nauk Armenia, Yerevan, 1983 (in Russian).

[29] R.W. James, The Optical Principles of the Diffraction of X-Rays, G. Bell and Sons, London, 1958.

[30] T. Shintake, Nucl. Instrum. Methods A 311 (1992) 453. 